\title{
Digestibility of new dietary fibre materials, resistant glucan and hydrogenated resistant glucan in rats and humans, and the physical effects in rats
}

\author{
Tsuneyuki Oku ${ }^{1,2 *}$, Kenichi Tanabe ${ }^{2,3}$, Shigeki Morita ${ }^{2}$, Norihisa Hamaguchi ${ }^{4}$, Fumio Shimura ${ }^{1}$ and \\ Sadako Nakamura ${ }^{1}$ \\ ${ }^{1}$ Laboratory of Nutrition, Institute of Food, Nutrition and Health, Jumonji University, 2-1-28 Sugasawa, Niiza, Saitama \\ 352-8510, Japan \\ ${ }^{2}$ Department of Nutritional Sciences, Graduate School of Human Health Science, University of Nagasaki Siebold, 1-1-1 \\ Manabino, Nagayo, Nagasaki 851-2195, Japan \\ ${ }^{3}$ Department of Food Science and Nutrition, Nagoya Women's University, 3-40, Shioji, Mizuho-ku, Nagoya 467-8610, Japan \\ ${ }^{4}$ Nibon Shokubin Kako Co. Ltd, 30 Tajima, Fuji 417-8530, Japan
}

(Submitted 20 January 2015 - Final revision received 24 May 2015 - Accepted 13 July 2015)

\section{Abstract}

Resistant glucan (RG) and hydrogenated resistant glucan (HRG) are newly developed non-digestible carbohydrate materials that decrease lifestyle-related diseases. The bioavailability of RG and HRG was investigated by in vitro experiments using human and rat small intestinal enzymes and by in vivo experiments using rats in the present study. Oligosaccharides, which are minor components of RG and HRG, were hydrolysed slightly by small intestinal enzymes of humans and rats, and the hydrolysing activity was slightly higher in rats than in humans. The amount of glucose released from HRG was greater than that from RG. However, the high-molecular-weight carbohydrates of the main components were hardly hydrolysed. Furthermore, neither RG nor HRG inhibited disaccharidase activity. When rats were raised on a diet containing $5 \%$ of RG, HRG, resistant maltodextrin or fructo-oligosaccharide (FOS) for 4 weeks, all rats developed loose stools and did not recover during the experiment, except for the FOS group. Body weight gain was normal in all groups and was not significantly different compared with the control group. Caecal tissue and content weights were significantly increased by feeding RG or HRG, although other organ and tissue weights were not significantly different among the groups. In conclusion, RG and HRG consist of small amounts of glucose and digestible and non-digestible oligosaccharides, and large amounts of glucose polymers, which were hardly hydrolysed by $\alpha$-amylase and small intestinal enzymes. RG and HRG, which were developed newly as dietary fibre materials, had no harmful effects on the growth and development of rats.

Key words: Digestibility: Physical effects: New dietary fibre material: Resistant glucan: Hydrogenated resistant glucan

Non-digestible carbohydrates such as dietary fibre and non-digestible oligosaccharides have health benefits and have been reported to prevent metabolic syndrome and lifestylerelated diseases ${ }^{(1-3)}$. However, the intake of non-digestible carbohydrate is gradually decreasing in developed countries, and the incidence of chronic diseases such as diabetes mellitus, heart disease and obesity is increasing ${ }^{(4)}$. To increase the intake of non-digestible carbohydrates, convenient food ingredients that can be easily added to meals and processed foods should be developed, contributing to preventing chronic diseases.

Some non-digestible carbohydrate materials with distinctive properties have been developed and are widely used in meals and processed food. Polydextrose (PD), developed as a low-energy bulking carbohydrate material, is used in many beverages and low-energy foods ${ }^{(5,6)}$. Resistant maltodextrin
(RMD) is another dietary fibre material used in many foods and was developed to suppress blood glucose elevation by carbohydrate foods and to improve defecation ${ }^{(7-9)}$. Fructooligosaccharide (FOS) is a non-digestible oligosaccharide present in many foods that was developed as a low-energy sweetener and non-cariogenic sweetener ${ }^{(9-11)}$. Non-digestible oligosaccharides including FOS are also used as prebiotics to improve intestinal microflora ${ }^{(12,13)}$.

Resistant glucan (RG) and hydrogenated resistant glucan (HRG) are newly developed non-digestible carbohydrate materials, which were made from starch hydrolysates ${ }^{(14)}$, as well as PD and RMD. RG is a white odourless powder that is easily dissolved in cold water and can be widely used as a low-energy bulking carbohydrate material. HRG is produced from RG by hydrogenation and is more stable than RG towards

Abbreviations: BBMV, brush border membrane vesicles; FOS, fructo-oligosaccharide; HRG, hydrogenated resistant glucan; PD, polydextrose; RG, resistant glucan; RMD, resistant maltodextrin.

* Corresponding author: T. Oku, fax +81484789367, email t-oku@jumonji-u.ac.jp 
degradation by the Maillard reaction ${ }^{(14)}$. Non-digestible low-energy bulking carbohydrate materials such as PD and RMD have been already used in meals and foods to prevent metabolic syndrome and lifestyle-related diseases ${ }^{(5-9)}$. However, the metabolic pathway and physiological function of RG and HRG have not been reported yet. We have hypothesised that the digestibility and metabolic pathway of RG and HRG are similar to those of PD and RMD, because they also consist of monosaccharide, disaccharide, oligosaccharides and glucose polymers.

Therefore, the aim of the present study was to assess the potential of these new dietary fibre materials as food ingredients that could eventually contribute towards preventing metabolic syndrome and lifestyle-related diseases. To elucidate the objective, the metabolic pathways and physiological functions of RG and HRG were achieved as follows: (1) by observing the hydrolysis of RG and HRG using small intestinal enzymes of humans and rats and comparing them with RMD; (2) by measuring the inhibitory effect of RG and HRG using disaccharidases from rat small intestine; (3) by observing physical effects such as growth, gastrointestinal tract and other organ weights, blood biochemical parameters and faecal shape on rats raised on a diet containing RG or HRG for 4 weeks.

\section{Methods}

\section{Ethical approval of the study protocol}

The protocol for animal studies (approval no. 2011-16) was approved by the Committee on Animal Experiments of the University of Nagasaki (Nagasaki, Japan). These experiments were conducted according to the Guidelines on the Care and Use of Laboratory Animals (National Institutes of Health) and the standards relating to the Care and Management of Experimental Animals (Notification number 88, from the Prime Minister's Office).

Experiments using donated tissues from human intestines were conducted according to guidelines laid down in the
Declaration of Helsinki. All procedures involving human subjects were approved by the Ethical Committee of the University of Nagasaki Siebold (received number 22, approval number 19) and Juzenkai Hospital in Nagasaki, Japan. All subjects provided written informed consent to participate in the study. All experiments were carried out in the Public Health Nutrition Laboratory of the Graduate School of Human Health Science, University of Nagasaki Siebold.

\section{Materials}

RG (dietary fibre content, $82 \%$; Nihon Shokuhin Kako Co. Ltd) was developed by heating glucose at $180^{\circ} \mathrm{C}$ in the presence of activated $\mathrm{C}^{(14)}$. In addition to its ability to act as a catalyst in the polycondensation of saccharides, activated $\mathrm{C}$ provides added benefits by being easily separable from the reactants and thus avoiding discolouration of the product. RG is a mixture of monosaccharides, oligosaccharides and glucose polymers with an average molecular weight of approximately $2600 \mathrm{Da}$, and the expected structure is shown in Fig. $1^{(14)}$. Purified RG (dietary fibre content, $99 \%$; Nihon Shokuhin Kako Co. Ltd), from which mono- and di-saccharides had been removed from RG, was used to investigate the digestibility and physiological function of core components of RG in the present study, and HRG (dietary fibre content, $82 \%$; Nihon Shokuhin Kako Co. Ltd), was also used to compare with the digestibility and physiological function of RG. The dietary fibre content of RG and HRG was measured by the enzymatic-HPLC method of the Association of Official Analytical Chemists (AOAC) 2001.03 method $^{(15)}$.

RMD (Fibresol-2; Matsutani Chemical Industry Co. Ltd) and FOS (Meiji Co. Ltd, formerly-Meiji Seika Kaisha Ltd) were used to compare with RG and HRG in the present study. RMD has been established as a dietary fibre material and was used as a positive control. RMD is a mixture of monosaccharides, oligosaccharides and glucose polymers containing $92 \%$ dietary fibre by the AOAC 2001.03 method. Its average molecular weight is about

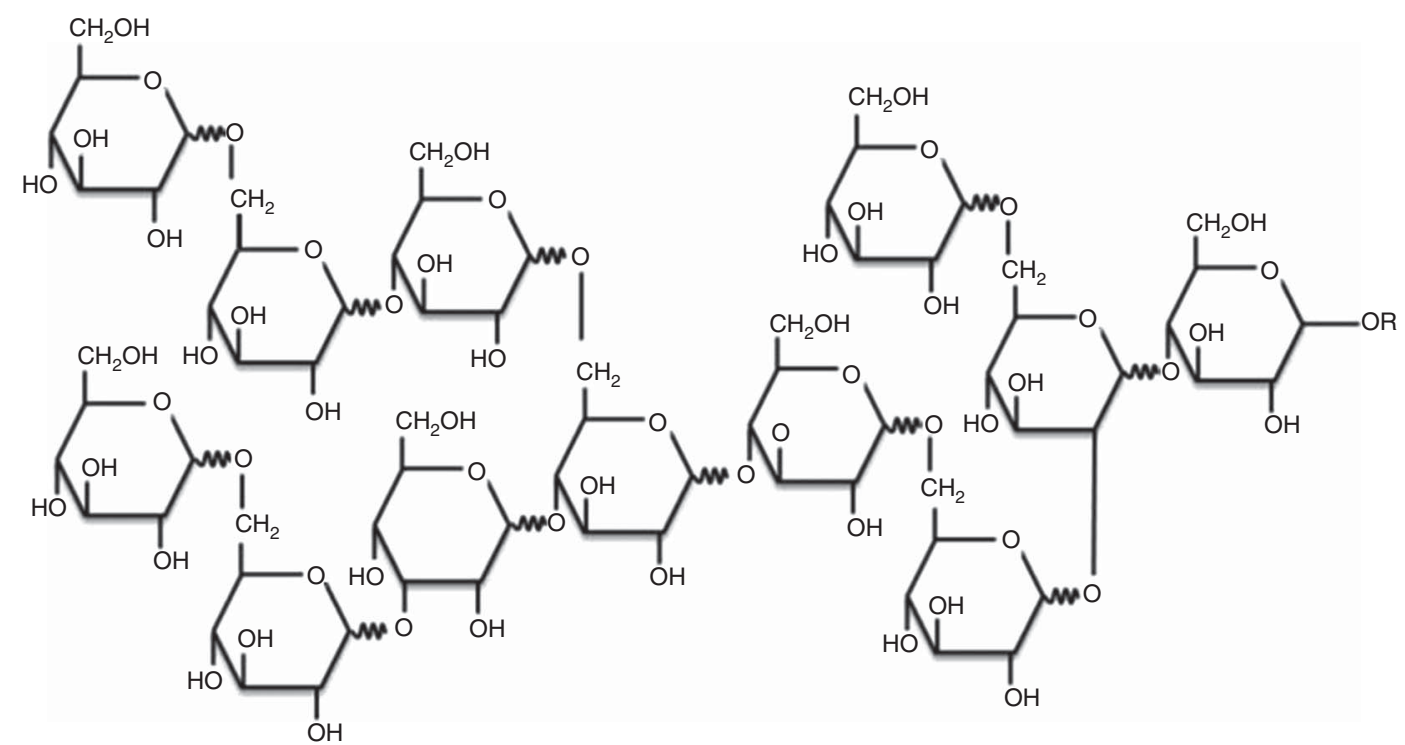

Fig. 1. Expected chemical structure of resistant glucan. 
2000 Da. FOS (purity; 98\%) was used as a typical non-digestible oligosaccharide that is not digested by small intestinal enzymes and is fermented completely by intestinal microbes. FOS is a mixture of 1-kestose $(36 \cdot 8 \%)$, nystose $(51.9 \%), 1 \mathrm{~F}-\beta$-fructofranosyl-nystose $(9 \cdot 3 \%)$ and monosaccharide and disaccharide (2\%).

\section{Hydrolysis of resistant glucan and hydrogenated resistant glucan using brush border membrane vesicles and homogenates of rat small intestine}

Animals. To prepare brush border membrane vesicles (BBMV) and homogenates of the small intestine, twenty rats (Wistar male, $320 \mathrm{~g}$ body weight; Clea Japan Inc.) were fed a standard solid diet (MF diets; Oriental Yeast Co.) and distilled water ad libitum for $5 \mathrm{~d}$. The animal quarters were air-conditioned at a temperature of $22-24^{\circ} \mathrm{C}$. Relative humidity was controlled at $50-55 \%$, and the rats were maintained on a $12 \mathrm{~h}$ light-dark cycle (08.00-20.00 hours, light; 20.00-08.00 hours, dark).

\section{Preparation of homogenates from rat small intestinal} mucosa

After overnight fasting, five rats were euthanised by decapitation. The small intestines were immediately removed, slit open, and washed with ice-cold $0.9 \% \mathrm{NaCl}$. The mucosa were scraped off with glass slides onto an ice-chilled glass plate, weighed, and homogenised with nine volumes of ice-cold $0.9 \% \mathrm{NaCl}(10 \%$ wet w/v) using a homogeniser (Polytron; Kinematica Inc.). All homogenates were stored at $-80^{\circ} \mathrm{C}$ until assayed. Before the assay, they were re-homogenised and diluted to the required concentration.

\section{Preparation of brush border membrane vesicles from rat small intestinal mucosa}

Small intestinal BBMV from fifteen male Wistar rats (final body weight $350 \mathrm{~g}$ ) were prepared by the modified method of Kessler et al. $^{(16)}$. The BBMV obtained were suspended in an adequate volume of $0.9 \% \mathrm{NaCl}$ and stored at $-80^{\circ} \mathrm{C}$ until assayed. Before the assay, they were re-homogenised and diluted to the required concentrations.

\section{Hydrolysis of resistant g/ucan and hydrogenated resistant glucan using rat small intestinal brush border membrane vesicles}

RG and HRG were hydrolysed following the method of Oku et al. ${ }^{(17)}$ partially modified by the Dahlqvist method using glucose oxidase et al. ${ }^{(18)}$. Glucose was used as a standard. RG and HRG as substrates were prepared at $100 \mathrm{mg} / \mathrm{ml}(38.5 \mathrm{~mm}$, mean molecular weight $2600 \mathrm{Da}$ ) in $0 \cdot 1 \mathrm{~m}$-maleate-Na buffer ( $\mathrm{pH} 6 \cdot 0$ ). Sucrose prepared at $112 \mathrm{~mm}$ in $0 \cdot 1 \mathrm{~m}$-maleate-Na buffer ( $\mathrm{pH} 6 \cdot 0$ ) was used as a control.

Diluted BBMV $(0.1 \mathrm{ml})$ were pipetted into a small glass test tube and then $0 \cdot 1 \mathrm{ml}$ of $100 \mathrm{mg} / \mathrm{ml} \mathrm{RG}$ or HRG or $112 \mathrm{~mm}$-sucrose was added to the test tube after preincubation for a few minutes at $37^{\circ} \mathrm{C}$. After incubation for a further $10-30 \mathrm{~min}$ at $37^{\circ} \mathrm{C}, 2.4 \mathrm{ml} \mathrm{TGO}$ reagent, containing $10 \mathrm{U} / \mathrm{ml}$ glucose oxidase, $5 \mathrm{U} / \mathrm{ml}$ peroxidase, $50 \mathrm{~mm}$-4-aminoantipyrine and $400 \mathrm{~mm}$ - $p$-phenolsulfonic acid $\mathrm{Na}$ salt in $0.5 \mathrm{M}-$ Tris- $\mathrm{HCl}(\mathrm{pH} 7 \cdot 0)$, was added to the test tube to stop the hydrolysis. The glucose oxidase and peroxidase reactions started simultaneously. The reaction mixture was further incubated for $10 \mathrm{~min}$ at $37^{\circ} \mathrm{C}$. Thereafter, two drops of $4 \mathrm{~N}-\mathrm{NaOH}$ were added to the test tube to stop the reactions, and the absorbance was read at a wavelength of $500 \mathrm{~nm}$ using a spectrophotometer (UVmini-1240; Shimadzu Corp.). The amount of glucose produced was calculated as hydrolysing activity ( $\mu \mathrm{mol}$ of glucose produced/mg protein per $\mathrm{h}$ ).

Hydrolysis of resistant glucan and hydrogenated resistant glucan using human and rat small intestinal homogenates

Preparation of human small intestinal homogenate. Fragments of human small intestines with no malignant tissues were donated by five patients from the Juzenkai Hospital in Nagasaki, Japan. Patients provided written informed consent to donate a fragment of small intestine after resection of tumorous tissue from the small intestine. Immediately after resection, tissue fragments were soaked in ice-cold $0.9 \% \mathrm{NaCl}$ and transferred to our laboratory. Normal fragments with no lesions were cut and washed with ice-cold $0.9 \% \mathrm{NaCl}$. Three of the five fragments were a part of the lower ileum and two were from the lower jejunum. The mucosa was gently scraped off with two slide glasses onto an ice-chilled glass plate and homogenised in a 19-fold volume of ice-cold $0.9 \%$ $\mathrm{NaCl}$ using a homogeniser (Polytron; Kinematica Inc.) ${ }^{(19)}$ and stored at $-80^{\circ} \mathrm{C}$ until use.

\section{Hydrolysis of resistant glucan and hydrogenated resistant glucan using human and rat small intestinal homogenates}

To compare the digestibility of RG and HRG, hydrolysis was carried out using human and rat small intestinal homogenates. Diluted homogenate $(0.1 \mathrm{ml})$ was pipetted into a small glass test tube and then $0.1 \mathrm{ml}$ of $100 \mathrm{mg} / \mathrm{ml} \mathrm{RG}$ or $H R G$ in $0 \cdot 1$ M-maleate-Na buffer ( $\mathrm{pH}$ 6.0) was added after preincubation for a few minutes at $37^{\circ} \mathrm{C}$. The assay followed the same hydrolysis procedure as described above.

\section{Hydrolysis of resistant glucan and hydrogenated resistant glucan using human salivary amylase}

Saliva was collected from two healthy human subjects into an Erlenmeyer flask using a triangular funnel with filter paper. The saliva collected was filtrated with a sterile filter $(0.22 \mu \mathrm{m}$; Millipore Corp.). $\alpha$-Amylase activity was determined using the Caraway method $^{(20)}$ and the activity was calculated as follows: $\alpha$-amylase activity $=\left(E_{\mathrm{B} 1}-E \mathrm{~s}\right) / E_{\mathrm{B} 1} \times 800 . E_{\mathrm{B} 1}$ is the absorbance without saliva and $E_{\mathrm{S}}$ is the absorbance with saliva.

\section{Inhibitory effect of resistant glucan and hydrogenated resistant glucan on sucrase and maltase using brush border membrane vesicles of rat small intestine}

To observe the inhibitory effect of RG and HRG on sucrase and maltase, 0, 2, 10, 50 and $100 \mathrm{mg} / \mathrm{ml}$ solutions of RG and HRG were prepared using $0 \cdot 1 \mathrm{M}$-maleate-Na buffer ( $\mathrm{pH}$ 6.0) as previously described $^{(21)}$. After $0 \cdot 1 \mathrm{ml}$ of the diluted BBMV was pipetted into a small glass test tube, $20 \mu \mathrm{l}$ of RG or HRG solutions was added to 
the test tube after preincubation for a few minutes at $37^{\circ} \mathrm{C}$, and the same volume of $0 \cdot 1 \mathrm{~m}$-maleate-Na buffer ( $\mathrm{pH}$ 6.0) was added as a control. Thereafter, $0.1 \mathrm{ml}$ of $112 \mathrm{~mm}$ substrate (sucrose or maltose) was added to the test tube. After incubation for $10-30 \mathrm{~min}$ at $37^{\circ} \mathrm{C}$, $2.4 \mathrm{ml}$ TGO reagent was added to the test tube. The assay followed the inhibitory assay procedure described above.

\section{Determination of protein concentration}

The protein concentrations of the BBMV and homogenates were determined using the Bradford method ${ }^{(22)}$. Bovine serum albumin was used as a standard.

\section{Consecutive feeding of rats using diets containing resistant} glucan or hydrogenated resistant glucan

Animals and diets. A total of twenty-five Wistar male rats (4 weeks old; Clea Japan Inc.) were fed a control diet for $3 \mathrm{~d}$ and then assigned to one of five groups ( $n 5$ per group) and fed as follows for 4 consecutive weeks. The control diet was an AIN93G diet containing $10 \%$ sucrose $^{(23)}$ with the cellulose being replaced with $\beta$-maize starch to exclude the effect of cellulose on intestinal microflora. In the initial experimental protocol, diets containing $10 \%$ RG or HRG had been planned for use in the present study. However, immediately after the control diet was switched to diets containing $10 \% \mathrm{RG}, \mathrm{HRG}$ or RMD at the end of the adaptation period of $3 \mathrm{~d}$, all rats developed loose stools. Therefore, the experimental diets were replaced with $5 \%$ of RG, HRG or RMD instead of $\beta$-maize starch in the AIN93G diet in the present study. The FOS diet consisted of a modified AIN93G diet in which $5 \%$ sucrose had been replaced by FOS. Rats were housed in individual stainless steel cages.

\section{Feeding conditions, animal care, observation of faecal shape and diet efficiency}

The feeding of the diet was restricted slightly to facilitate equal food intake. Food and drinking water intake and body weight were measured on alternate days, and the amount of diet was adjusted to maintain normal body weight gain. Health status and faecal conditions were observed daily to monitor for loose stools. The definition of loose stools was very soft faeces and was classified according to six categories ${ }^{(24,25)}$. Diet efficiency for the experiment period was calculated by body weight gain divided by the total food intake. Blood $(120 \mu \mathrm{l})$ was collected several times from the rat's tails using a haematocrit tube during the experimental period. Serum prepared by haematocrit centrifugation was used for glucose and insulin assays. After the rats were fasted for $16 \mathrm{~h}$ at the end of the experiment, they were euthanised by decapitation and blood was collected and centrifuged (H-3R; Kokusan Co. Ltd) at $2000 \mathrm{~g}, 20^{\circ} \mathrm{C}$, for $15 \mathrm{~min}$ to obtain serum. The serum was used for the assay of glucose, insulin, TAG and other biochemical parameters.

\section{Measurement of organ and adipose tissue weights and small and large intestinal lengths}

Immediately after opening the rat's abdomen, the liver, kidney, spleen, lung, heart, testis and epididymal and perirenal adipose tissues were removed and organ weights were measured. After the stomach and the small and large intestines were cut open along the mesenteric side, they were washed with ice-cold $0.9 \% \mathrm{NaCl}$, blotted with paper and weighed. After the total weight of the caecum and contents was measured, the caecal content was removed and the tissue was weighed.

\section{Assay of serum glucose and insulin}

The glucose concentration of the serum was measured by the Trinder method ${ }^{(26)}$ using glucose oxidase. After serum $(10 \mu \mathrm{l})$ was pipetted into a small test tube, glucose oxidase reagent $(1.5 \mathrm{ml})$ was added and the test tube was incubated at $37^{\circ} \mathrm{C}$ for $15 \mathrm{~min}$. The test tube was then steeped in boiling water for $150 \mathrm{~s}$ to stop the reaction. The absorbance was read with a spectrophotometer (UVmini-1240) at a wavelength of $505 \mathrm{~nm}$. The insulin concentration was measured immunologically with the ELISA kit (Morinaga Biochemical Institute Co.) using guinea pig antibodies ${ }^{(27)}$.

Measurement of free fatty acid, TAG, total protein, albumin, aspartic aminotransferase and alanine aminotransferase

Several blood biochemical parameters were measured with assay kits (Wako Chemical Co.) as follows: total cholesterol, cholesterol E test Wako (GPD/DAOS method); TAG, TAG E test Wako (GPD/DAOS method); free fatty acid, NEFA test Waco (ACS/ACOD method); total protein and albumin, A/GB test Wako (BCG/Buret method); and aspartate transaminase and alanine transaminase and transaminase CII test Wako (POP/ TOOS method).

\section{Calculations and statistical analyses}

The activity of disaccharidase using rat intestinal BBMV and human small intestinal homogenate was calculated as specific activity ( $\mu \mathrm{mol}$ of substrate hydrolysed/mg protein per $\mathrm{h}$ ), and mean values and standard deviations were calculated for duplicate assays and then compared with inhibitors at the same concentration using the Student $t$ test. For feeding experiments, data were calculated as mean values and standard deviations and compared with the control group by ANOVA and Dunnett's post hoc test. A $P$ value $<5 \%$ was considered significant using SPSS version 21 .

\section{Results}

In vitro hydrolysing experiments

Hydrolysis of resistant glucan and hydrogenated resistant glucan using brush border membrane vesicles of rat small intestine. When RG and HRG were incubated with BBMV of rat small intestine at $37^{\circ} \mathrm{C}$, glucose released from HRG was much more than that from RG (Table 1). RG released glucose $(328 \mu \mathrm{g} /$ $\mathrm{mg}$ protein per $\mathrm{h})$ and sucrose released glucose $(984 \mu \mathrm{g} / \mathrm{mg}$ protein per $\mathrm{h}$ ). The amount of glucose released from HRG was $1056 \mu \mathrm{g} / \mathrm{mg}$ protein per h, noticeably more than from RG from which disaccharides had been removed. Thus, RG and HRG might be partially used as an energy source when ingested by humans. 
Table 1. Hydrolysis of resistant glucan (RG) and hydrogenated resistant glucan (HRG) by brush border membrane vesicles of rat small intestine*

\begin{tabular}{lcc}
\hline Substrates & $\begin{array}{c}\text { Produced glucose } \\
(\mu \mathrm{g} / \mathrm{mg} \text { of protein } / \mathrm{h})\end{array}$ & $\begin{array}{c}\text { Relative ratio } v . \\
\text { sucrose }\end{array}$ \\
\hline $\mathrm{RG}$ & 328 & 0.33 \\
$\mathrm{HRG}$ & 1056 & 1.07 \\
Sucrose & 984 & 1.00 \\
\hline
\end{tabular}

* Assay condition: $100 \mu \mathrm{l}$ of substrate $(100 \mathrm{mg} / \mathrm{ml}$ of RG or HRG or $112 \mathrm{~mm}$ of sucrose) and $100 \mu \mathrm{l}$ of enzyme were incubated at $37^{\circ} \mathrm{C}$ for $15-30 \mathrm{~min}$, and the produced glucose was measured at optical density $500 \mathrm{~nm}$ by the colorimetric assay using glucose oxidase.

\section{Comparison of resistant glucan and hydrogenated resistant glucan hydrolysis using human and rat small intestinal homogenates}

When RG and HRG were incubated with human small intestinal homogenates at $37^{\circ} \mathrm{C}$, glucose was released from both RG and HRG as well as rat small intestinal homogenate, and the amount of glucose released was much more from from RG by rat small intestinal homogenate was approximately $33 \%$ of that of sucrose, and the amount of glucose released from HRG was similar to that of sucrose. However, the amount of glucose released from RG by human small intestinal homogenate was approximately $22 \%$ of that of sucrose, and the amount of glucose released from HRG was $<67 \%$ of that of sucrose. The hydrolysis of RG and HRG was relatively lower in humans than in rats based on the hydrolysis of sucrose. These results suggest that the hydrolysis of RG and HRG is more resistant to human small intestinal enzymes than those from rats.

\section{Inhibitory effect of resistant glucan and hydrogenated} resistant glucan for disaccharidases using brush border membrane vesicles of rat small intestine

The inhibitory effect of RG and HRG on sucrase and maltase was observed using BBMV of rat small intestine. As shown in Table 2, RG and HRG did not inhibit sucrase activity, but rather the amount of glucose released increased depending on the concentration of RG and HRG. From a colorimetric assay of inhibitory effect, the absorbance of the blank and the net amount of glucose produced also increased depending on the concentration of RG and HRG. The amount of glucose produced was greater for HRG than for RG. These results suggest that RG and HRG contain some glucose and glucose polymers that are partially used as a substrate. In addition, RG and HRG did not inhibit maltase activity either (data not shown).

\section{Hydrolysis of resistant glucan and hydrogenated resistant glucan using human salivary amylase}

Although the molecular weight of RG and HRG is low (approximately $2600 \mathrm{Da}$ ), these molecules might be hydrolysed by $\alpha$-amylase. The hydrolysing activity of $\alpha$-amylase for RG and HRG was measured by a colorimetric assay using the iodine reaction (Table 3). The absorbance of RG and HRG not treated

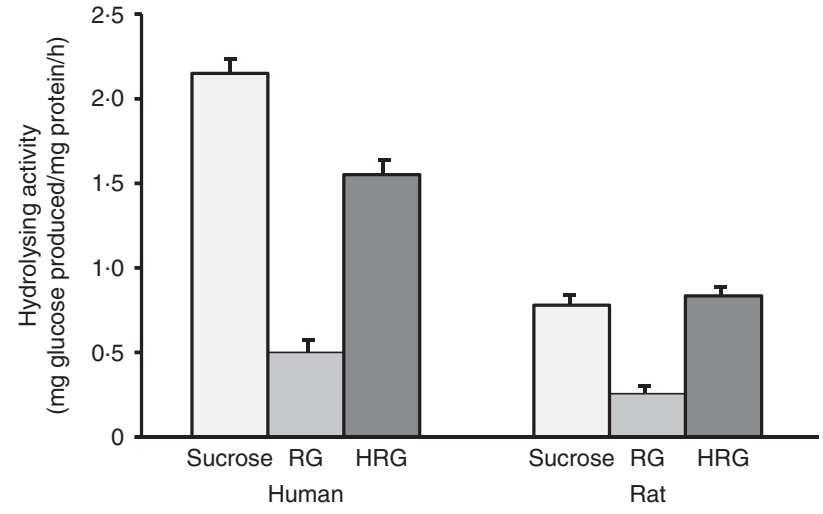

Fig. 2. Hydrolysis of resistant glucan (RG) and hydrogenated resistant glucan (HRG) by human and rat small intestinal homogenates. Assay condition: $100 \mu \mathrm{l}$ of substrate and $100 \mu \mathrm{l}$ of enzyme were incubated at $37^{\circ} \mathrm{C}$ for $15-30 \mathrm{~min}$ and the glucose produced was measured at optical density $500 \mathrm{~nm}$ by the colorimetric assay using glucose oxidase. Values are means with standard deviations represented by vertical bars.

Table 2. Inhibitory effect of resistant glucan (RG) purified and hydrogenated resistant glucan (HRG) on sucrase of brush border membrane vesicles (BBMV) of rat small intestine*

\begin{tabular}{cccc}
\hline $\begin{array}{c}\text { Concentration } \\
\text { of inhibitor } \\
(\mathrm{mg} / \mathrm{ml})\end{array}$ & $\begin{array}{c}\text { Apparent glucose } \\
\text { produced }(\mu \mathrm{g} / \\
200 \mu \mathrm{l})\end{array}$ & $\begin{array}{c}\text { Glucose in } \\
\text { blank }(\mu \mathrm{g} / \\
200 \mu \mathrm{l})\end{array}$ & $\begin{array}{c}\text { Net produced } \\
\text { glucose }(\mu \mathrm{g} / 15 \mathrm{~min} \\
\text { per } 200 \mu \mathrm{l})\end{array}$ \\
\hline $\mathrm{RG}$ & & & \\
0 & 249 & 0 & 249 \\
2 & 250 & 6 & 244 \\
10 & 256 & 9 & 247 \\
50 & 287 & 20 & 267 \\
100 & 299 & 25 & 274 \\
$\mathrm{HRG}$ & & & 249 \\
0 & 249 & 0 & 260 \\
2 & 266 & 6 & 266 \\
10 & 286 & 20 & 324 \\
50 & 368 & 44 & 384 \\
100 & 434 & 50 & \\
\hline
\end{tabular}

* Assay condition: $100 \mu \mathrm{l}$ of $112 \mathrm{~mm}$ of sucrose, $20 \mu \mathrm{l}$ of RG or HRGM and $100 \mu \mathrm{l}$ of BBMV of rat small intestine were incubated at $37^{\circ} \mathrm{C}$ for $15-30 \mathrm{~min}$, and the produced glucose was measured at optical density $500 \mathrm{~nm}$ by the colorimetric assay using glucose oxidase.

Table 3. Hydrolysis of resistant glucan (RG) purified and hydrogenated resistant glucan (HRG) by human salivary $a$-amylase*

\begin{tabular}{lc}
\hline Substrates $(100 \mathrm{mg} / \mathrm{ml})$ & Activity $(\mathrm{U})$ \\
\hline Soluble starch & 5560 \\
RG & 320 \\
HRG & 0 \\
RMD & 216 \\
\hline
\end{tabular}

RMD, resistant maltodextrin.

* Assay condition: $1 \mathrm{ml}$ of $0.25 \mathrm{~m}$ of substrate and $0.1 \mathrm{ml}$ of diluted saliva were mixed and incubated at $37^{\circ} \mathrm{C}$ for $7.5 \mathrm{~min}$. Then it stood in boiled water to stop the reaction for $5 \mathrm{~min}$. After $1 \mathrm{ml}$ of dye solution was added, the optical density was read at $660 \mathrm{~nm}$.

with $\alpha$-amylase was very weak, similar to that for RMD, but was high for soluble starch (positive control). Thus, RG and HRG were hardly hydrolysed by human salivary $\alpha$-amylase, whereas soluble starch was decomposed spontaneously. These results demonstrate that RG and HRG are not hydrolysed by $\alpha$-amylase when ingested by humans. 
Consecutive feeding of rats using diets containing resistant glucan or hydrogenated resistant glucan

Effects of feeding resistant glucan and hydrogenated resistant glucan on the growth and osmotic loose stools of rats. Immediately after the control diet was switched to diets containing $5 \%$ RG, HRG or RMD, all rats developed loose stools, which remained during the 4 weeks of experiments. The shape of the loose stools was no different among the RG, HRG and RMD groups, despite differences in the content of non-digestible oligosaccharides in the three groups. The FOS group also developed loose stools immediately after switching to the $5 \%$ FOS diet, but recovered within 1 week and faeces returned to their normal shape.

Fig. 3 shows the growth curves of rats during the experimental period. Body weight gain was normal and the final body weight was not significantly different among the five groups, despite loose stools throughout the experimental period. Thus, RG and HRG did not harm the growth and development of rats.

Effect of resistant glucan and hydrogenated resistant glucan feeding on food intake, body weight gain and diet efficiency

The food intake, diet efficiency and body weight gain were slightly greater in the control group compared with the other four groups, but were not significantly different (Table 4).

\section{Effect of resistant glucan and hydrogenated resistant glucan} feeding on weight of organ and tissue

Table 5 shows the weight of organs and tissues per $100 \mathrm{~g}$ of body weight, except for the caecum and colon. The weight of (Mean values and standard deviations) some organs was slightly different among the five groups, but no differences based on diet composition were observed among the five groups, including the control group. The weights of adipose tissues were not different among the five groups (Fig. 4).

\section{Effect of resistant glucan and hydrogenated resistant glucan} feeding on gastrointestinal tract and caecal content weight

The weight of caecal tissue and content was greatly increased by feeding with RG and HRG for 4 weeks (Fig. 5). The weight of caecal tissue in RG, HRG, RMD and FOS groups significantly

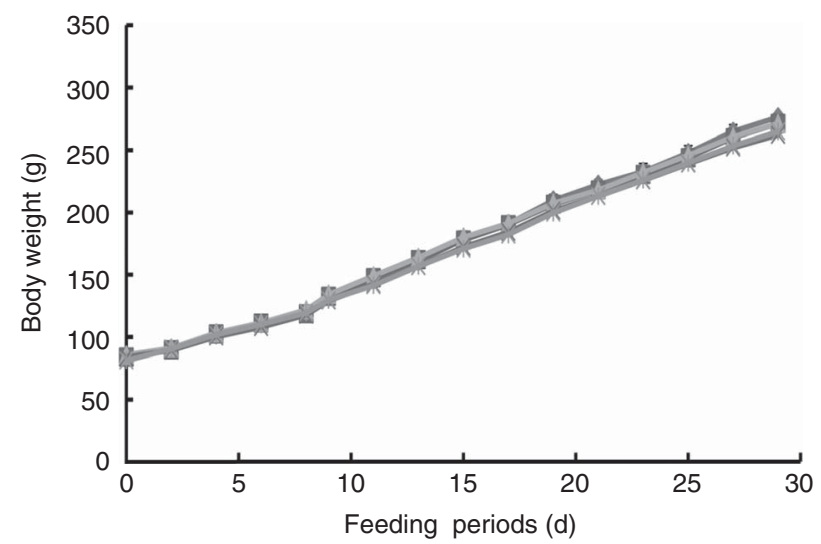

Fig. 3. Growth curves of rats fed resistant glucan (RG) and hydrogenated resistant glucan (HRG). Diets were AIN93G with $\beta$-maize starch replaced by $5 \%$ of RG, HRG, RMD or FOS. No significant difference was observed among groups. FOS, fructo-oligosaccharide; RMD, resistant maltodextrin. _ _ _, $5 \%$ RG; - - , control; $-\Delta_{-}, 5 \%$ HRG; $-X-, 5 \%$ RMD; _ * _, $5 \%$ FOS.

Table 4. Body weight, total intake and diet efficiency in rats fed diet containing resistant glucan (RG) purified or hydrogenated resistant glucan (HRG)*

\begin{tabular}{|c|c|c|c|c|c|c|c|c|c|c|c|c|}
\hline \multirow[b]{3}{*}{ Groups } & \multicolumn{6}{|c|}{ Body weight (g) } & \multicolumn{4}{|c|}{ Total intake (g) } & & \\
\hline & \multicolumn{2}{|c|}{ Initial } & \multicolumn{2}{|c|}{ Final } & \multicolumn{2}{|c|}{ Gain } & \multicolumn{2}{|c|}{ Diet } & \multicolumn{2}{|c|}{ Water } & \multicolumn{2}{|c|}{ Diet efficiency (\%) } \\
\hline & Mean & SD & Mean & SD & Mean & SD & Mean & SD & Mean & SD & Mean & SD \\
\hline Control & $85 \cdot 3$ & $2 \cdot 8$ & 276.4 & 11.7 & $191 \cdot 1$ & $10 \cdot 0$ & $532 \cdot 6$ & 4.8 & 1032 & 138 & 35.9 & 1.8 \\
\hline $5 \%$ RG & 84.0 & 2.5 & 271.2 & 7.0 & $187 \cdot 2$ & 4.7 & $529 \cdot 2$ & 7.6 & 965 & 171 & $35 \cdot 4$ & 0.5 \\
\hline $5 \% \mathrm{HRG}$ & $85 \cdot 8$ & 2.5 & $271 \cdot 1$ & $8 \cdot 1$ & $185 \cdot 3$ & $8 \cdot 3$ & 524.8 & $20 \cdot 4$ & 1029 & 106 & $34 \cdot 3$ & 1.8 \\
\hline $5 \%$ RMD & $84 \cdot 2$ & $4 \cdot 3$ & $262 \cdot 1$ & $11 \cdot 6$ & $178 \cdot 0$ & $11 \cdot 6$ & 518.5 & $8 \cdot 7$ & 1065 & 178 & $34 \cdot 3$ & $2 \cdot 6$ \\
\hline $5 \%$ FOS & $81 \cdot 2$ & 4.0 & 264.8 & $6 \cdot 1$ & 183.6 & $3 \cdot 8$ & $509 \cdot 2$ & $18 \cdot 1$ & 794 & 114 & $36 \cdot 1$ & 1.1 \\
\hline
\end{tabular}

FOS, fructo-oligosaccharide; RMD, resistant maltodextrin.

* Body weight gain, total intake of diet and water, and diet efficiency were not significantly different among the five groups.

Table 5. Weight of organs and tissues of rats fed a diet containing resistant glucan (RG) purified or hydrogenated resistant glucan (HRG) (Mean values and standard deviations)

\begin{tabular}{|c|c|c|c|c|c|c|c|c|c|c|c|c|c|c|c|c|}
\hline \multirow[b]{2}{*}{ Groups } & \multicolumn{2}{|c|}{ Heart } & \multicolumn{2}{|c|}{ Lungs } & \multicolumn{2}{|c|}{ Kidneys } & \multicolumn{2}{|c|}{ Liver } & \multicolumn{2}{|c|}{ Spleen } & \multicolumn{2}{|c|}{ Testis } & \multicolumn{2}{|c|}{ Stomach } & \multicolumn{2}{|c|}{ Small intestine } \\
\hline & Mean & SD & Mean & SD & Mean & SD & Mean & SD & Mean & SD & Mean & SD & Mean & SD & Mean & SD \\
\hline Control & 0.31 & 0.01 & 0.38 & 0.03 & 0.70 & 0.04 & 3.64 & 0.14 & 0.26 & 0.03 & 1.03 & 0.10 & 0.42 & 0.05 & 1.99 & 0.11 \\
\hline $5 \%$ RG & 0.31 & 0.01 & 0.37 & 0.02 & 0.69 & 0.02 & 4.01 & 0.52 & 0.24 & 0.02 & 1.07 & 0.04 & 0.37 & 0.02 & 2.02 & 0.13 \\
\hline $5 \% \mathrm{HRG}$ & 0.29 & 0.01 & 0.38 & 0.02 & 0.70 & 0.03 & 3.38 & 0.31 & 0.24 & 0.02 & 1.08 & 0.06 & 0.39 & 0.01 & 2.03 & 0.12 \\
\hline $5 \%$ RMD & 0.30 & 0.02 & 0.41 & 0.05 & 0.68 & 0.03 & 3.29 & 0.05 & 0.25 & 0.01 & 0.99 & 0.05 & 0.37 & 0.02 & 2.06 & 0.13 \\
\hline $5 \%$ FOS & 0.32 & 0.02 & 0.40 & 0.02 & 0.69 & 0.05 & 3.93 & 0.22 & 0.27 & 0.01 & 1.09 & 0.04 & 0.39 & 0.02 & $2 \cdot 19$ & 0.18 \\
\hline
\end{tabular}

FOS, fructo-oligosaccharide; RMD, resistant maltodextrin. 

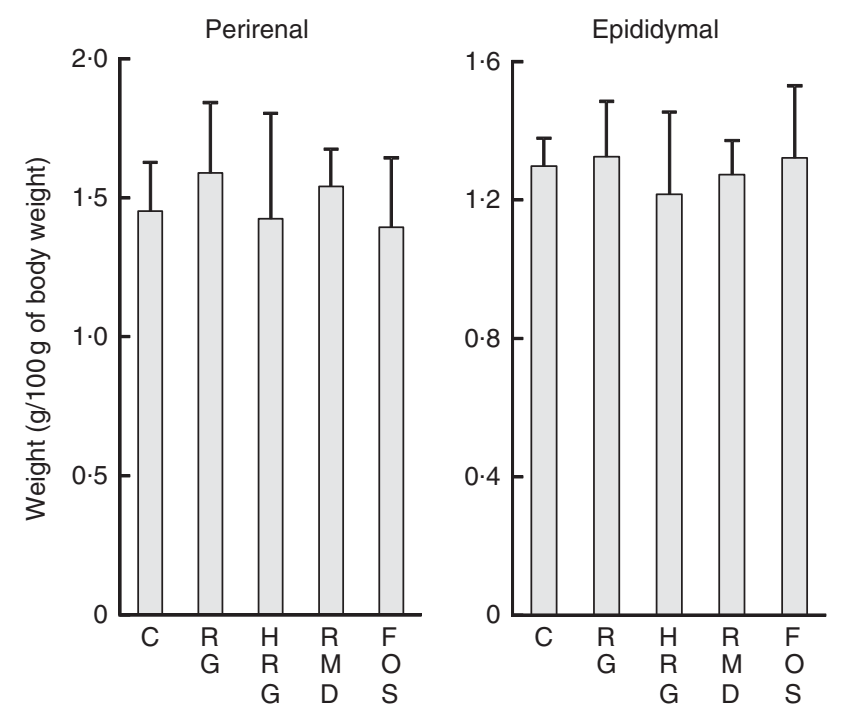

Fig. 4. Weight of adipose tissues in rats fed a diet containing resistant glucan (RG) or hydrogenated resistant glucan (HRG). Diets were AIN93G in which $\beta$-maize starch was replaced with $5 \%$ of RG, HRG, RMD or FOS. Values are means and standard deviations. No significant difference was observed among groups. C, control; FOS, fructo-oligosaccharide; RMD, resistant maltodextrin.

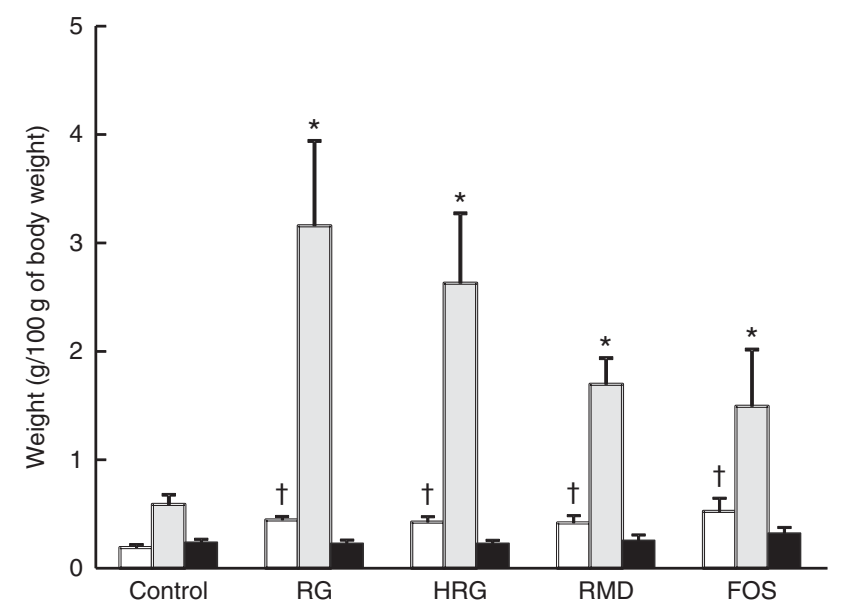

Fig. 5. Weight of caecal contents, tissues of caecum and those of colon in rats fed a diet containing resistant glucan (RG) or hydrogenated resistant glucan (HRG). Diets were AIN93G in which $\beta$-maize starch was replaced with $5 \%$ of $R G$, HRG, RMD or FOS. Values are means with standard deviations represented by vertical bars. $\dagger,{ }^{*}$ Significantly different $v$. control diet group, respectively, at $P<0.05$ by ANOVA and Dunnett's post hoc test. RMD, resistant maltodextrin; FOS, fructo-oligosaccharide. $\square$, Caecal tissues; , caecal contents; $\square$, colon. increased compared with the controls $(P<0 \cdot 05)$. The weight of caecal content in the RG and HRG groups was five times that of the control group and was greater in the RG group than in the HRG group. The weight of caecal content was also significantly higher in the RMD and FOS groups but was lower than in the RG and HRG groups. The weight of the stomach and small intestines was not significantly different among the five groups, including the control group (Table 5).

\section{Effect of resistant glucan and hydrogenated resistant glucan feeding on blood biochemical parameters}

The concentration of metabolites was normal in the RG, HRG, RMD, FOS and control groups with no significant differences between the five groups (Table 6).

\section{Discussion}

To investigate the digestibility of RG and HRG, their hydrolysis was observed using BBMV of rat small intestine and then compared with those of human small intestinal homogenates. At the same time, the inhibitory effect of RG and HRG was measured on disaccharidases from the rat small intestine. Furthermore, rats were raised on diets containing RG or HRG for 4 weeks, and the physical effects on growth, gastrointestinal tract and other organ weights, blood biochemical parameters and faecal shape were measured.

The enzymatic-HPLC method of AOAC $2001.03^{(15)}$ is the Official Method of Analysis for determining the dietary fibre content of food products. Using this method, purified RG contained $99 \%$ of dietary fibre and HRG was $82 \%$. Thus, purified RG contained $1 \%$ digestible carbohydrate and HRG contained $18 \%$ of digestive carbohydrate. In this method, $\alpha$-amylase and amyloglucosidase from Aspergillus niger, whose hydrolysing activity is stronger than that of rats, were used to hydrolyse carbohydrates in the food product. Therefore, the results imply that $99 \%$ of purified RG is resistant to hydrolysis by glucosidase because $>99 \%$ of RG is not digested by the small intestinal enzymes of humans and rats. In the present study, the remaining $1 \%$ of RG was hydrolysed by small intestinal enzymes and a small amount of glucose was released. As $18 \%$ of HRG was hydrolysed by $\alpha$-amylase and amyloglucosidase, $18 \%$ of HRG was digested by small intestinal enzymes and the amount of glucose released from HRG increased noticeably compared with RG. These results demonstrate that purified RG is a

Table 6. Effects of feeding a diet containing resistant glucan (RG) purified or hydrogenated resistant glucan (HRG) on biochemical parameters in serum* (Mean values and standard deviations; five rats)

\begin{tabular}{|c|c|c|c|c|c|c|c|c|c|c|c|c|c|c|c|c|c|c|}
\hline \multirow[b]{2}{*}{ Groups } & \multicolumn{2}{|c|}{ Glucose (mmol/l) } & \multicolumn{2}{|c|}{ Insulin (mg/l) } & \multicolumn{2}{|c|}{ T-chol (mg/l) } & \multicolumn{2}{|c|}{ TAG $(g / l)$} & \multicolumn{2}{|c|}{ NEFA (mEq/l) } & \multicolumn{2}{|c|}{$\mathrm{TP}(\mathrm{g} / \mathrm{l})$} & \multicolumn{2}{|c|}{ Alb (g/l) } & \multicolumn{2}{|c|}{ AST (U/I) } & \multicolumn{2}{|c|}{ ALT (U/l) } \\
\hline & Mean & $\mathrm{SD}$ & Mean & SD & Mean & $\mathrm{SD}$ & Mean & $\mathrm{SD}$ & Mean & SD & Mean & $\mathrm{SD}$ & Mean & SD & Mean & SD & Mean & SD \\
\hline Control & $5 \cdot 5$ & 0.1 & 423 & 35 & 1.9 & 0.2 & 1.04 & 1.4 & 0.9 & 0.0 & 50 & 5 & 33 & 1 & 22 & 7 & 15 & 7 \\
\hline $5 \% R G$ & $5 \cdot 1$ & 0.1 & 516 & 34 & $2 \cdot 0$ & 0.3 & $1 \cdot 20$ & 1.8 & 0.9 & 0.3 & 55 & 7 & 33 & 0 & 20 & 13 & 17 & 11 \\
\hline $5 \%$ HRG & $5 \cdot 1$ & 0.1 & 428 & 16 & 1.8 & 0.1 & $1 \cdot 14$ & 0.5 & 0.9 & 0.2 & 53 & 4 & 31 & 2 & 18 & 9 & 18 & 7 \\
\hline $5 \%$ RMD & 4.9 & 0.1 & 429 & 53 & $2 \cdot 0$ & 0.2 & 1.14 & 0.5 & 0.9 & 0.1 & 49 & 7 & 30 & 1 & 21 & 14 & 15 & 5 \\
\hline $5 \%$ FOS & $5 \cdot 7$ & 0.1 & 531 & 42 & 1.9 & 0.2 & 1.06 & 1.6 & 1.0 & 0.5 & 51 & 5 & 32 & 0 & 23 & 15 & 19 & 11 \\
\hline
\end{tabular}

T-chol, total cholesterol; TP, total protein; Alb, albumin; AST, aspartate transaminase; ALT, alanine transaminase; RMD, resistant maltodextrin; FOS, fructo-oligosaccharide.

* The concentrations of metabolites such as glucose, insulin, T-chol, TAG, NEFA, TP, Alb, AST and ALT were not significantly different among the five groups. 
resistant carbohydrate that is barely hydrolysed by small intestinal enzymes and $\alpha$-amylase, and that HRG is a resistant carbohydrate containing a small amount of carbohydrate that is only slightly hydrolysed by small intestinal enzymes.

The present study also suggests that oligosaccharide, a minor component of RG and HRG, was hydrolysed and glucose was released. The amount of glucose released from HRG was significantly more than that from RG with $99 \%$ of dietary fibre. Furthermore, the amount of glucose released from RG and HRG increased depending on the concentration of RG and HRG in the experiments on the inhibition of sucrase and maltase. If glucose polymers in RG and HRG as well as oligosaccharide, a minor component, are hydrolysed spontaneously by small intestinal enzymes, glucose should be produced actively and thus the amount of glucose released from both RG and HRG must be the same. However, the amount of glucose released from RG was $33 \%$ of that of sucrose, and that from HRG was almost the same as that of sucrose. The reason for the amount of glucose released from RG being less than that from HRG seems to be related to the amount of oligosaccharide, not to the amount of glucose polymer in RG and HRG. These results suggest that the oligosaccharide fraction of $R G$ and $H R G$ is readily hydrolysed by the small intestinal enzymes of rats but glucose polymers are not or are barely hydrolysed. The results obtained for dietary fibre content are reasonable for RG and HRG.

The amount of glucose released from HRG was equal to that from sucrose, and the amount of glucose released from RG was approximately $33 \%$ of that of sucrose. However, the digestibility of RG and HRG cannot be simply evaluated from these results, because the concentration of the substrate was markedly different from that of sucrose, and the chemical structure of sucrose as a substrate differs greatly from that of RG or HRG. These results suggest that oligosaccharides in RG and HRG were preferentially hydrolysed by small intestinal enzymes because the oligosaccharide content as the substrate of small intestinal enzymes is abundant in HRG, from which small-molecular-weight saccharides have not been removed, compared with RG, from which they have been removed. If the high-molecular-weight carbohydrates in RG and HRG are easily hydrolysed by small intestinal enzymes, the amount of glucose released from RG must increase, as well as the amount of sucrose. In addition, these results indicate that the amount of glucose released from HRG, which contains abundant oligosaccharides, is greater than that from purified RG, and suggest that the glucose measured is derived from oligosaccharides, and it is not from high-molecular-weight carbohydrates of the main component in RG and HRG.

Hydrogenated sugar alcohols such as maltitol and lactitol are more resistant to hydrolysis by disaccharidases compared with maltose and lactose, which are their precursors ${ }^{(19,28,29)}$. Sugar alcohols with a degree of polymerisation $>2$ are slowly hydrolysed by disaccharidases compared with disaccharide alcohol. HRG, which is RG that has been hydrogenated, was readily hydrolysed compared with purified RG, and much more glucose was produced. The probable reason for this was that maltopolyols, such as maltitol and maltotritol contained in HRG, had been hydrolysed slowly by maltase $\mathrm{e}^{(30)}$.
RG and HRG were also hydrolysed by small intestinal enzymes of humans, although their hydrolysing activity was slightly different from those of the rat small intestinal enzymes. The hydrolysing activity for RG compared with that of sucrose was $<22 \%$ using human small intestinal enzyme and approximately $33 \%$ using rat small intestinal enzyme. Meanwhile, the hydrolysing activity for HRG compared with that for sucrose was slightly less (approximately $67 \%$ ) in humans and similar in rats. These results indicate that RG and HRG are more resistant to hydrolysis in humans than in rats based on the hydrolysis of sucrose. Furthermore, the fact that the hydrolysing activity by small intestinal enzymes is lower in humans than in rats has also been observed for highly cross-linked $\operatorname{starch}^{(31)}$, whereas the digestibility of oligosaccharides, such as sucrose and maltose, is similar in both humans and rats ${ }^{(19)}$.

RG, HRG and RMD were hardly hydrolysed by human salivary $\alpha$-amylase. Thus, they may not be used as substrates of $\alpha$-amylase, because their mean molecular weight is about $2600 \mathrm{Da}$, which is too small for $\alpha$-amylase. In addition, $\alpha$-amylase may not be able to break down the glucoside linkage of the high-molecular-weight carbohydrates in RG and HRG. Thus, these high-molecular-weight carbohydrates in RG and HRG are not hydrolysed by digestive enzymes such as $\alpha$-amylase and disaccharidase, although some oligosaccharides in RG and HRG are hydrolysed by small intestinal enzymes. These results are not inconsistent with the dietary fibre content of RG and HRG.

Some monosaccharides (L-arabinose and D-sorbose) and oligosaccharides (acarbose) inhibit the activity of hydrolysing enzymes such as sucrase and maltase ${ }^{(27,28)}$. Therefore, RG and HRG may inhibit the activity of disaccharidase, because they are synthesised from starch hydrolysate at random. However, in the present study, RG and HRG did not inhibit the activity of sucrase and maltase using BBMV from rat small intestines. In preference, when the concentration of RG and HRG as inhibitors was increased from 0 to $100 \mathrm{mg} / \mathrm{ml}$, the amount of glucose released from RG and HRG increased depending on the concentration used. Besides, the absorbance of the blank also increased depending on the concentration of RG and HRG. These results demonstrate that RG and HRG contain a small amount of glucose and oligosaccharide that is hydrolysed by small intestinal enzymes. Therefore, glucose originally contained and glucose released from oligosaccharide in RG and HRG might increase blood glucose and insulin levels when humans ingest RG and HRG.

Our preliminary experiment used a diet containing $10 \% \mathrm{RG}$ or HRG for consecutive feeding of rats. Immediately after the rats started the diet containing $10 \%$ RG, HRG or RMD, very loose stools developed in all rats. Therefore, the diets were changed immediately to contain $5 \%$ RG, HRG or RMD. However, loose stools remained until the end of the experiment. Loose stools are considered to be caused by increased osmotic pressure induced by small-molecular-weight saccharides. Although the diet containing $5 \%$ of FOS also caused loose stools, this stopped within 1 week and faeces returned to the normal shape. Rats given large amounts of non-digestible oligosaccharides such as FOS, lactulose and galactosylsucrose developed loose stools that stopped within 1 or 2 weeks, with faeces returning to normal ${ }^{(25)}$. One explanation for this is that intestinal microbes that readily use non-digestible oligosaccharides increased spontaneously and quickly used the 
oligosaccharides. In the present study, loose stools following feeding by RG and HRG remained until the end of the experiment. These results demonstrate that intestinal microbes that readily use RG and HRG did not proliferate. Indirectly, this supports the view that RG and HRG are resistant to fermentation by intestinal microbes. If RG and HRG are fermented spontaneously by intestinal microbes, hydrogen would be produced and excreted. Therefore, we would like to measure hydrogen excretion in further experiments using rats and healthy human subjects.

PD, made artificially from monosaccharides, causes very loose stools that do not recover, as well as RG and $\mathrm{HRG}^{(32,33)}$. Thus, RG, HRG and PD, made artificially from starch hydrolysates, seem to have a specific chemical structure that is resistant to digestive enzymes and intestinal microbes. Therefore, RG and HRG are likely to have low bioavailability in humans.

Body weight gain was normal in all groups given the test diet and in the control group, although diet feeding was restricted slightly and osmotic loose stools developed. In addition, the blood biochemical parameters were not significantly different among the five groups. Thus, RG and HRG had no harmful effects on the growth and development of rats. The weight of caecal tissue and content increased greatly after feeding RG or HRG, although the weight of other organs and tissues was not significantly different among all groups. Specific increments in caecal tissue and content weights have been clearly observed by feeding on non-digestible carbohydrates such as dietary fibre, non-digestible oligosaccharide and sugar alcohol ${ }^{(25,34,35)}$. Therefore, the phenomenon of caecal tissue enlargement seems to be one of adaptation to non-digestible carbohydrates. In addition, the weight of caecal content was greater for RG than for HRG, and that for RMD was lower than that for HRG. These results suggest that $R G$ is the most resistant to digestion and fermentation of three materials, and that the amount of RG undigested that arrives at the caecum is the greatest of the three test substances.

Purified RG, which mono- and di-saccharides had been removed from RG, was slightly hydrolysed by the small intestinal enzymes of rats and humans, and glucose was released distinctly from oligosaccharides in RG. However, the high-molecularweight carbohydrate that is included in RG and HRG was not hydrolysed by disaccharidase and $\alpha$-amylase. Furthermore, loose stools, whose incidence depends on the amount of non-digestible oligosaccharides, were triggered by feeding RG. These results demonstrate that RG contains both digestible and non-digestible oligosaccharides. Also, the amount of glucose released from HRG, which is RG that has been hydrogenated, was greater than that from RG. Therefore, HRG must contain an abundant amount of digestible and non-digestible oligosaccharides compared with RG. These results agree with the dietary fibre content of RG and HRG products.

In conclusion, RG and HRG contained small amounts of digestible and non-digestible oligosaccharides and large amounts of glucose polymers that were hardly hydrolysed by $\alpha$-amylase and small intestinal enzymes, and had no harmful effects on the growth and development of rats. Therefore, RG and HRG could be used as new dietary fibre materials.

\section{Acknowledgements}

The authors thank Nihon Shokuhin Kako Co. Ltd (Tokyo, Japan) for providing RG purified and HRG. They also thank the Matsutani Chemical Industry Co. Ltd (Hyogo, Japan) for providing RMD, and the Meiji Co. Ltd (formerly-Meiji Seika Kaisha Ltd, Tokyo, Japan) for providing FOS.

This study was supported in part by Grant-in-Aid for Scientific Research (B) (2) 22300263 and university funding for research to $\mathrm{T}$. O.

T. O. designed the research and had primary responsibility for the final content. T. O. and S. N. conducted the research, analysed the data and performed the statistical analysis. K. T, S. M. and F. S. conducted the research and analysed the data. N. H. contributed by making the RG and HRGM. All authors read and approved the final manuscript.

The author(s) have declared the following potential conflicts of interest with respect to the research, authorship and/or publication of this article. T. O., S. N., T. K., S. M. and F. S. have no conflicts of interest. N. H. is a member of staff of the Institute of Nippon Shokuhin Kako Co. Ltd and made the resistant glucan and hydrogenated resistant glucan.

\section{References}

1. Oba S, Nagata C, Nakamura K, et al. (2010) Dietary glycemic index, glycemic load, and intake if carbohydrate and rice in relation to risk of mortality from stroke and its subtypes on Japanese men and women. Metabolism 59, 1574-1582.

2. Ross AB, Bruce SJ, Blondel-Lubrano A, et al. (2011) A Whole-grain cereal-rich diet increases plasma betain and tends to decrease total and LDL-cholesterol compared with a refined-grain diet in healthy subjects. Br J Nutr 105, 1492-1502.

3. Kim Y \& Je Y (2014) Dietary fibre intake and total mortality; a meta-analysis of prospective cohort studies. Am J Epidemiol 180, 565-573.

4. Rahati S, Shahraki M, Arijomand G, et al. (2014) Food pattern, lifestyle and diabetes mellitus. Int J High Risk Behav Addict $\mathbf{3}$, e8725.

5. Auerbach MH, Craig SAS, Howlett JF, et al. (2007) Caloric availability of polydextrose. Nutr Rev 65, 544-549.

6. Konings E, Schoffelen PF, Stegen J, et al. (2013) Effect of polydextrose and soluble maize fibre on energy metabolism, metabolic profile and appetite control in overweight men and women. Br J Nutr 107, 1-11.

7. Bear DJ, Stote KS, Henderson T, et al. (2014) The metabolizable energy of dietary maltodextrin is variable and alters fecal microbiota composition in adult men. $J$ Nutr 144, 1023-1029.

8. Hashizume C, Kishimoto Y, Kanamori S, et al. (2012) Improvement of resistant maltodextrin in humans with metabolic syndrome by continuous administration. J Nutr Sci Vitaminol 58, 423-430.

9. Yamada K, Sato-Mito N, Nagata J, et al. (2008) Health claim evidence requirements in Japan. J Nutr 138, Suppl., s1192-s1198.

10. Eslamparast T, Zamani F, Hekmatdoost A, et al. (2014) Effect of symbiotic supplementation on insulin resistance in subjects with metabolic syndrome: a randomized, double-blind, placebo-controlled pilot study. Br J Nutr 112, 438-445.

11. Respondek F, Hipipre C, Chouveau P, et al. (2014) Digestive tolerance and postprandial glycemic and inslinaemic responses after consumption of dietary desserts containing maltitol and fructooligosaccharides in adults. Eur J Clin Nutr 68, 575-580. 
12. Roberfroid M (2007) Prebiotics: the concept revisited. J Nutr 137, Suppl. 2, 830S-837S.

13. Mitsuoka T (2014) Development of functional foods. Biosci Microbiota Food Health 33, 117-128 (in Japanese).

14. Hamaguchi N, Hirai H, Aizawa K, et al. (2014) Production of water-soluble indigestible polysaccharides using activated carbon. J Appl Glycosci 61, 100-115.

15. Ohkuma K, Matsuda I, Katta Y, et al. (2000) New method for determining total dietary fiber by liquid chromatography. J AOAC Int 83, 1013-1019.

16. Kessler M, Acuto O, Strelli C, et al. (1978) A modified procedure for the rapid preparation of efficiently transporting vesicles from small intestinal brush border membranes. Biochem Biophys Acta 506, 136-154.

17. Oku T, Konishi F \& Hosoya N (1982) Mechanism of inhibitory effect of unavailable carbohydrate on intestinal calcium absorption. J Nutr 112, 410-415.

18. Dahlqvist A (1976) Method of assay of intestinal disaccharidases. Anal Biochem 7, 18-25.

19. Oku T, Tanabe K, Ogawa S, et al. (2011) Similarity of hydrolyzing activity of human and rat small intestinal disaccharidases. Clin Exp Gastroenterol 4, 155-161.

20. Caraway WT (1959) A stable starch substrate for the determination of amylase in serum and other body fluids. Am J Clin Path 32, 97-99.

21. Oku T, Yamada M, Nakamura M, et al. (2006) Inhibitory effects of extractives from leaves of Morus alba on human and rat small intestinal disaccharidase activity. Br J Nutr 95 , 933-938.

22. Bradford MM (1976) A rapid and sensitive method for the quantitation of microgram quantities of protein utilizing the principle of protein-dye binding. Anal Biochem 72, 248-258.

23. Reeves PG, Nielsen FH \& Fahey GC Jr (1993) AIN-93 purified diets for laboratory rodents: final report of the American Institute of Nutrition ad hoc writing committee on the reformulation of the AIN-76A rodent diet. J Nutr 123, 1939-1951.

24. Nakamura S \& Oku T (2002) Replicability of the effect of galactosylsucrose-containing food for specified health uses on fecal improvement in the case of availableness on usual life. JJpn Assoc Dietary Fibre Res 6, 73-80 (in Japanese).
25. Tanabe K, Nakamura S \& Oku T (2008) Effects of non-digestible oligosaccharides with different properties on growth, osmotic diarrhea, lipid metabolism, fecal hydrolase activity and production of short chain fatty acids of caecal contents in rats. JJpm Assoc Dietary Fibre Res 12, 17-29 (in Japanese).

26. Trinder P (1969) Determination of blood glucose using an oxidase-peroxidase system with a non-carcinogenic chromogen. J Clin Pathol 22, 158-161.

27. Liversy JH, Hodgkinson SC, Round HR, et al. (1980) Effect of time, temperature and freezing on the stability of immunoreactive $\mathrm{LH}, \mathrm{FSH}, \mathrm{TSH}$, growth hormone, prolactin and insulin in plasma. Clin Biochem 13, 151-155.

28. Seri K, Sanai K, Matsuo N, et al. (1996) L-Arabinose selectively inhibits sucrase in an uncompetitive manner and suppressers glycemic response after sucrose ingestion in animals. Metabolism 45, 1368-1374.

29. Oku T, Murata-Takenoshita Y, Yamazaki Y, et al. (2014) Inhibitory effect of D-sorbose on the small intestinal disaccharidases of humans and rats, and suppressive effects upon postprandial blood levels of glucose and insulin by D-sorbose in rats. Nutr Res $\mathbf{3 4}$, 961-967.

30. Yoshizawa S, Moriuchi S \& Hosoya N (1975) The effects of maltitol on rat intestinal disaccharidases. J Nutr Sci Vitaminol 21, 31-39.

31. Tachibe M, Ohga H, Nishibata T, et al. (2011) Digestibility, fermentability, and energy value of highly cross-linked phosphate tapioca starch in men. J Food Sci 76, H152-H155.

32. Newberne PM, Conner MW \& Estes P (1988) The influence of food additives and related materials on lower bowel structure and function. Toxicol Pathol 16, 184-197.

33. Oku T, Okamatsu H \& Fujii Y (2000) Effect of polydextrose on fecal weight and gastrointestinal transit time in beagle dogs. JJpn Assoc Dietary Fibre Res 4, 29-35 (in Japanease).

34. Nakamura S, Kondo N, Yamaguchi Y, et al. (2014) Daily feeding of fructooligosaccharide or glucomannan delays onset of senescence in SAMP8 mice. Gastroenterol Res Prac 2014, article ID 303184.

35. Hongo R, Nakamura S \& Oku T (2010) Bioavailability of mannitol through intestinal microbes in rats. J Jpn Assoc Dietary Fibre Res 14, 13-22 (in Japanese). 